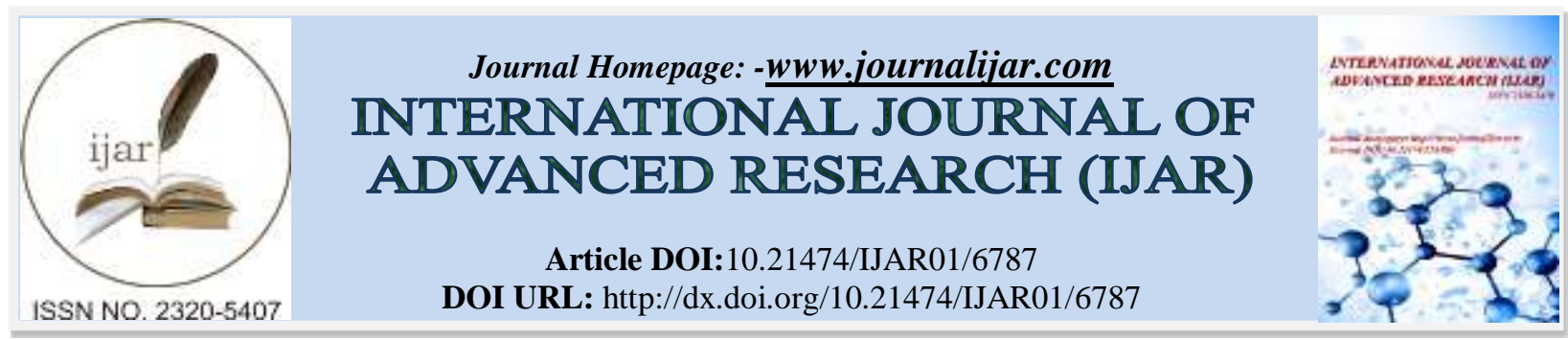

RESEARCH ARTICLE

\title{
DYNAMIC HYSTERESIS LOOP OF A FERROMAGNETIC LAYER: EFFECT OF THE HIGH SECOND ORDER OF THE ANISOTROPY CONSTANT.
}

A. Adanlété Adjanoh.

Département de Physique, Faculté des Sciences et Techniques, Université de Kara, B.P. 404, Kara-Togo.

\section{Manuscript Info}

Manuscript History

Received: 19 January 2018

Final Accepted: 21 February 2018

Published: March 2018

Keywords:-

Magnetization reversal; magnetic

anisotropy constant.

\section{Abstract}

Dynamic magnetization reversal measurements at room temperature have been performed by magneto-optical effect on $\mathrm{Au} / \mathrm{Co} / \mathrm{Au}$ sandwiches with perpendicular anisotropy. Domain wall displacement and domain nucleation regimes govern the magnetization reversal at low and high-applied field sweep rates, respectively. The transition between the two regimes occurs at $200 \mathrm{Oe} / \mathrm{s}$.

\section{Introduction:-}

The magnetization reversal dynamics in thin solid films with perpendicular anisotropy has been extensively studied during the last years for fundamental reason [1,2], as well as for magnetic recording applications $[3,4]$, where the reversal process of domain is inherently involved.

Direct magnetic observations by means of advanced magnetic imaging techniques [5] have shown that magnetization reversal in these systems occurs via two competing process: domain-nucleation and domain-wall motion. If the first is currently assumed to be thermally activated process with single [6] or distribution of energy barrier, the last can be described by thermally activated process or more complex viscous motions [7]. The predominance of one process against the others is governed by the variation rate of the magnetic applied field and the micro-structure of the material.

A lot of fundamental work have been devoted to the study of these processes in $\mathrm{Au} / \mathrm{Co} / \mathrm{Au}(111)$ sandwiches with perpendicular anisotropy for extended range of cobalt thickness $[2,8,9]$. It has shown that in sandwiches deposited in high vacuum chamber on several supports in accordance with standard techniques [10-12] the magnetization reversal is mainly controlled by domain motion initiated by a few micrometer size magnetic domain in the thickness range about 0.7 [13] $\mathrm{nm}$ where the magnetic anisotropy is relatively strong. In counterpart, the effect of a decrease of the anisotropy on the reversal magnetic process when one increases the magnetic field variation rate was not studied.

The aim of this paper is to investigate the influence of a reduction of the uniaxial anisotropy in $\mathrm{Au} / \mathrm{Co} / \mathrm{Au}$ sandwiches on the magnetization reversal dynamics.

Weaker anisotropic sandwiches are obtained by acting on the deposition process or using thin float glass supports to reduce the strains in the cobalt films, i.e. thermal strains as well as those induced by the lattice mismatch between cobalt and gold [14]. Anisotropy measurements from quasi-static response of three $\mathrm{Au} / \mathrm{Co} / \mathrm{Au}$ deposited on float glass are performed followed by a study of these hysteresis loops with field variation rate from quasi-static regime 
(12 Oe/s) to fast regime $(3750 \mathrm{Oe} / \mathrm{s})$. An interpretation of the magnetization reversal dynamics using two theoretical models is proposed flowing previous works $[9,15]$.

\section{Experimental procedures:-}

The Au/Co films are grown by electron beam evaporation on a float glass substrate after prior deposition of a $30 \mathrm{~nm}$ gold buffer layer at room temperature. This polycrystalline buffer is annealed at $450 \mathrm{~K}$ for one hour. Annealing increases the grain seize and smoothes the surface. We then obtained a well-textured polycrystalline film. The (111) close-packed planes on the face centered cubic structure of gold are preferentially parallel to the surface film and the lateral seize of the grains is about $100-200 \mathrm{~nm}$. At room temperature, cobalt grows epitaxially on this Au buffer with hcp (0001) structure. For each sample, the cobalt layer was deposited intermittently with an object to favor a good relaxation of the successive atomic plans. The relaxation favors the reduction of constraints and consequently minimizes the magneto-elastic contribution anisotropy. A $10 \mathrm{~nm}$ thick Au capping layer is finally deposited for protection. The magnetic properties of these films were measured, at room temperature, by Kerr magnetometer in polar configuration. Measurements are realized using a modulation technique of polarization state of the light.

\section{Results and discussion:-}

It is well known that for $\mathrm{Au} / \mathrm{Co} / \mathrm{Au}$ system magnetic anisotropy is perpendicular when cobalt thickness is below to critical thickness. Previous studies were shown that for our samples (prepared by electron beam evaporation) this critical thickness is about $1.7 \mathrm{~nm}$ [6]. Fig. 1 shows quasi-static (at a very weak sweep rate of the applied field) hysteresis loops with applied field perpendicular to the cobalt layer, for 1 and $1.5 \mathrm{~nm}$ cobalt thicknesses deposited on thick glass; the two systems are respectively called samples (a), (b) in all the work.
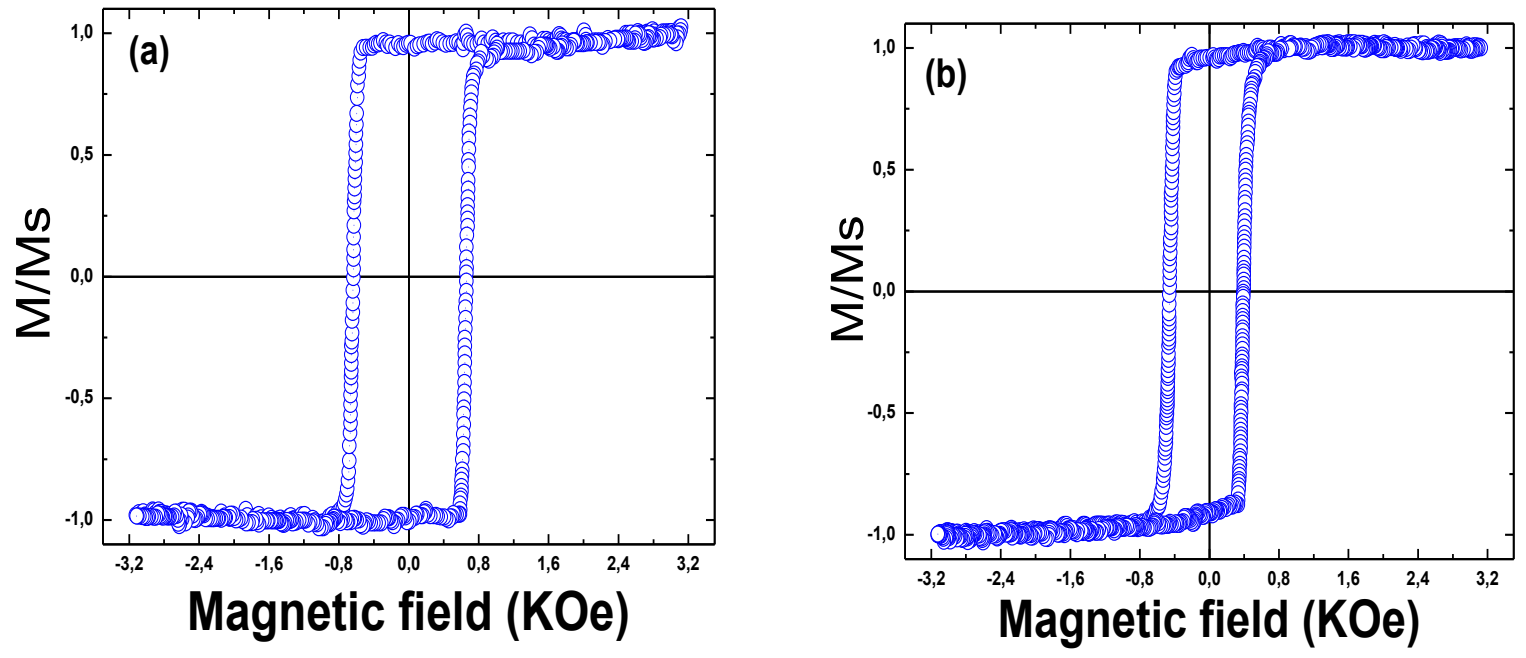

Fig. 1:-Quasi-static loops at room at $300 \mathrm{~K}$ of $\mathrm{Au} / \mathrm{Co} / \mathrm{Au}$ on thick glass. (a) $: \mathrm{t}_{\mathrm{Co}}=1 \mathrm{~nm}(\mathrm{~b}): \mathrm{t}_{\mathrm{Co}}=1.5 \mathrm{~nm}$.

Cobalt films exhibit a large remanence $\left(\frac{M r}{M_{s}}=1\right.$ for samples (a) and $\frac{M r}{M_{s}}=0.93$ for sample (b)) and a high coercivity (660 Oe for samples (a) and 390 Oe for sample (b)) indicating a strong perpendicular anisotropy. The abrupt reversal of magnetization from $+M_{s}$ to $-M_{s}$ at $-H_{C}$ value of applied field, shown in hysteresis loops, reflects that magnetization reversal via a slow initial nucleation process and a fast wall motion process [13]. Therefore the energy barrier for domain wall propagation is lower than that for nucleation.

Since the magnetization reversal process depends on the magnetic anisotropy, it is very important to have an idea on this property. Let us recall that the anisotropy energy of the Co hcp (0001) can be written as:

$$
E_{a}=K_{1} \sin ^{2} \theta+K_{2} \sin ^{4} \theta
$$


where $\theta$ is the angle between the magnetization and the surface normal, $K_{1}$ and $K_{2}$ are respectively the first-order and the second-order anisotropy constants. Generally, effect of $K_{2}$ on magnetization orientation is assumed to be lower than the one of $\mathrm{K}_{1}$. Hence, magnetization configuration depends drastically on magnitude of $K_{1}$ and on its sign. Then strong magnetic anisotropy corresponds to a higher positive $K_{1}$. The magnetic anisotropy constants can be determined by the measurement of the perpendicular magnetization component when the magnetic field is applied in a weaker $\alpha$ tilted direction from the in-plane direction (i.e. direction of the hard axis). Within a magnetic field range going from $H=0 \quad\left(M_{\perp}= \pm M_{s}\right)$ to $H= \pm H_{s}$ ( $H_{S}$ being the saturation field for which $\left.M_{\perp}=M_{s} \sin \alpha\right)$, a coherent rotation behavior is observed. Then the canting angle $\theta$ is given by $\cos \theta=\frac{M_{\perp}}{M_{s}}$.

The coherent rotation part of the loops can be fitted by:

$$
H=\frac{\left(2 K_{1} \sqrt{1-\cos ^{2} \theta}+4 K_{2}\left(1-\cos ^{2} \theta\right)^{3 / 2}\right) \cos \theta}{M_{s}\left(\cos \theta \cos \alpha-\sqrt{1-\cos ^{2} \theta} \sin \alpha\right)},
$$

deduced from the minimization of the energy anisotropy taking into account the Zeeman term. The fitting parameters were $\frac{2 K_{1}}{M_{s}}$ and $\frac{4 K_{2}}{M_{s}}$, the $K_{1}$ and $K_{2}$ values are shown in table I.

Table I:- Values of $K_{1}$ and $K_{2}$ obtained by fitting the coherent rotation parts of loops measured in tilted field.

\begin{tabular}{|c|c|c|}
\hline$t_{C o}(\mathrm{~nm})$ & 1 & 1.5 \\
\hline$K_{1}\left(10^{6} \mathrm{erg} / \mathrm{cm}^{3}\right)$ & $3.4 \pm 0.1$ & $1.04 \pm 0.14$ \\
\hline$K_{2}\left(10^{6} \mathrm{erg} / \mathrm{cm}^{3}\right)$ & $0.89 \pm 0.05$ & $0.52 \pm 0.08$ \\
\hline
\end{tabular}

We notes that, for the two samples, $K_{1}>0$ and $K_{2}>0$ describing an uniaxial magnetic anisotropy with an easy axis perpendicular to the cobalt film. Compared to $K_{1}, K_{2}$ is not negligible and its effect in the process of reversal magnetization should be considerable. For negligible $K_{2}$, in the case of a perpendicular anisotropy, magnetization reverses generally by propagation of wall domain. Whereas $K_{2}$ is so important enough the role of the nucleation process cannot be neglected. This effect is not very substantial in statics for sample (a) and more substantial for sample (b) since the hysteresis loop presents a delicate curvature. A method to probe reversal dynamics and to determine the relevant mechanism in the Co reversal process is to measure dynamics hysteresis loops and to examine the dynamic coercivity dependence of the field sweep rate. We measured the dynamic hysteresis loops (Fig.2) of the films on 2 decades of field sweep rate $\frac{d H}{d t}$, from $12 \mathrm{Oe} / \mathrm{s}$ to $3.75 \mathrm{KOe} / \mathrm{s}$ using the polar Kerr effect. 

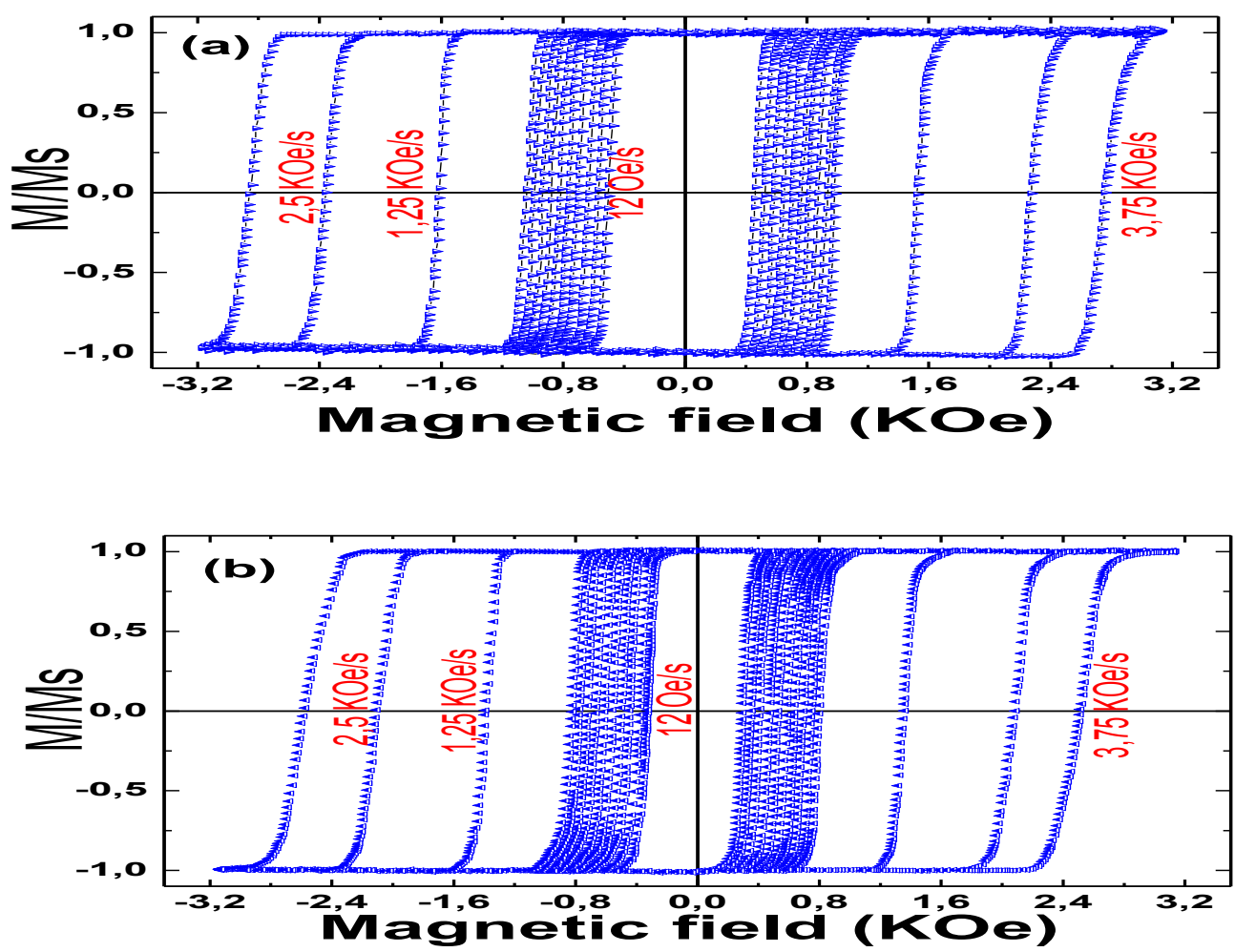

Fig.2:- Dynamic hysteresis loops for different values of dH/dt at 300K. (a) $\mathrm{Au} / \mathrm{Co}(1 \mathrm{~nm}) / \mathrm{Au}$, (b) : $\mathrm{Au} / \mathrm{Co}(1.5 \mathrm{~nm}) / \mathrm{Au}$.

Results of the figure 2 show that the widening of the loops of our three samples is very important for 2 decades of field sweep rate explored. This fast widening of the dynamic loops for these $\frac{d H}{d t}$ values is a new fact in comparison with the previous studies made on these kinds of systems [7], this could also be attribute to a probable purpose of remanence of the coil. However, the increase of the coercivities accompanied by widening transitions indicate that the magnetization reversal process is thermally activated.

Values of the dynamic coercive field Hc were extracted from the hysteresis loops and plotted as a function of field sweep rate $\frac{d H}{d t}$ (fig. 3) 

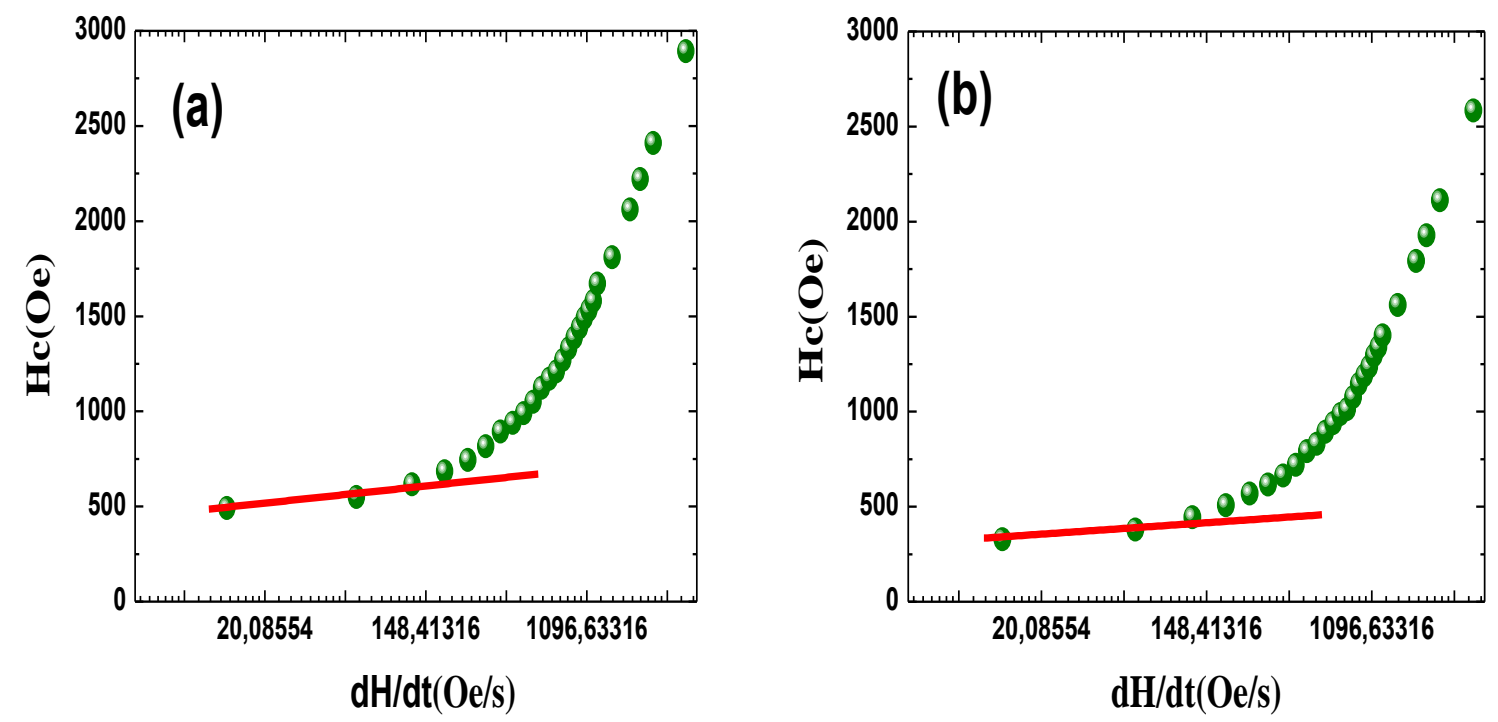

Fig. 3:- Coercivity dependence on $\ln (\mathrm{dH} / \mathrm{dt})$ of Co layers. (a) $\mathrm{Au} / \mathrm{Co}(1 \mathrm{~nm}) / \mathrm{Au}, \quad$ (b) $\mathrm{Au} / \mathrm{Co}(1.5 \mathrm{~nm}) / \mathrm{Au}$

There are two distinct regions in these data. First, there is the range up to $200 \mathrm{Oe} / \mathrm{s}$ where the dynamic coercive field Hc increases as a linear function of the logarithm of $\frac{d H}{d t}$. This behavior is consistent with the Bruno's model, which is based on domain wall dynamics [8]. Then the predominant mechanism seems to be domain wall propagation with weak pinning centers, defined by a specific relaxation time and activation energy linearly dependent on magnetic field. In this model Hc can be written, for a sweep rate given, like:

$$
H c=\frac{K_{B} T}{V_{B} M_{s}} \ln \left[\frac{V_{B} M_{s}}{K_{B} T} \ln (2) \tau(H=0) \frac{d H}{d t}\right] \quad,
$$

where $\mathrm{M}_{\mathrm{s}}$ is the saturation magnetization, $V_{B}$ is the characteristic volume which reverses magnetization during a wall jump called Barkhausen volume. $\tau(H=0)$ is the relaxation time at zero field.

Fitting data of this range by the expression (3) is presented on solid line on fig.4. From The fitting we deduced $\tau(\mathrm{H}=0)=3.71$ days and $\mathrm{V}_{\mathrm{B}}=6.47 \cdot 10^{-19} \mathrm{~cm}^{3}$ for sample (a) and $\tau(\mathrm{H}=0)=3.49$ days and $\mathrm{V}_{\mathrm{B}}=9.70 \cdot 10^{-19} \mathrm{~cm}^{3}$ for sample (b). The values of Barkhausen volume deduced from the fits are in the same magnitude order that those obtained by Raquet and al. [7] through their works done on $\mathrm{Au} / \mathrm{Co} / \mathrm{Au}$. On the other hand the $\tau(\mathrm{H}=0)$ values are weaker than those found in bibliography [9]. Since this last parameter describes the stability of the remanent magnetization, we see that these values are under estimated and not considered like absolute but relative because of the fact that the model used considers that the energy barrier is unique however it would not true in real layers. However the comparison of these values between them permits to affirm that $\tau(\mathrm{H}=0)$ decreases when magnetic anisotropy decrease. Second, there is the range beyond of $200 \mathrm{Oe} / \mathrm{s}$ where the dynamic coercivity diverges quickly with $\frac{d H}{d t}$. Here, domain nucleation plays a dominant role in the reversal. Nevertheless, the transition in the evolution of the coercive field according to $\frac{d H}{d t}$ is generally assigned to two possibilities: either a transition to a regime of pure nucleation or a transition from activated thermally wall motion to a viscous wall motion. 


\section{Conclusions:-}

In this work, the dynamic Coercivities of our layers reveal that the magnetization reversal by wall motion thermally activated becomes less importing very quickly to make places to another process where the nucleation dominated. Indeed, the sweep rate transition between the two regimes is around $200 \mathrm{Oe} / \mathrm{s}$, this value is relatively low in comparison with some magnitude orders found previously. This result can be explained by the relatively high value of $K_{2}$ called second order constant anisotropy; this observation could also be attribute to a probable purpose of remanence of the coil. One the other hand, that could be also explained by has high density of defect within the cobalt layer. We concluded thanks to our works that a square loop with an abrupt reversal does not present necessarily a dynamic of magnetization reversal dominated by a wall motion.

\section{Acknowledgments:-}

This work is the result of a collaboration between the LMOP laboratory (Tunisia) and the Université de Kara (Togo). We acknowledge the help of R. Belhi.

\section{References:-}

1. J. Ferré, J. P. Jamet, and P. Meyer, Phys. Status Solidi (a) 175, 213 (1999).

2. S.-B. Choe and S.-C. Shin, Phys. Rev. B 57, 1085 (1998); J. Appl. Phys. 83, 6952 (1998).

3. J. X. Shen, R. D. Kirby, Z. S. Shan, D. J. Sellmyer, and Tl Suzuki, J. Appl. Phys. 73, 6418 (1993).

4. J. Pommier, P. Meyer, G. Pénissard, J. Ferré, P. Bruno, and D. Renard, Phys. Rev. Lett. 65, 2054 (1990).

5. A. Kirilyuk, J. Ferré, V. Grolier, J. P. Jamet, and D. Renard, J. Magn. Magn. Mater. 171, 45 (1997); A. Kirilyuk, J. Ferré, J. Pommier, and D. Renard, J. Magn. Magn. Mater. 121, 536 (1993); A. Kirilyuk, J. Ferré, and D. Renard, IEEE Trans. Magn. 29, 2518 (1993).

6. R. Belhi, S. Jomni, N. Mliki, K. Abdelmoula, M. Ayadi, G. Clugnet, A. Charai, C. Leroux, G. Nihoul Can. J. Phys. 79(7): 1011-1020 (2001)

7. B. Raquet, M. D. Ortega, M. Goiran, A. R. Fert, J. P. Redoules, R. Mamy, J, C. Ousset, A. Sdaq, and A. Khmou. J. Magn. Magn. Mater. 150, L5 (1995). B. Raquet, R. Mamy, and J. C. Ousset, Phys. Rev B 54, 4128 (1996); J. Magn. Magn. Mater. 165, 492 (1997).

8. P. Bruno, G. Bayreuther, C. Chappert, G. Lugert, D. Renard, and J. Seiden, J. Appl. Phys. 68, 5759 (1990).

9. B. Raquet, M. D. Ortega, A. Sdaq, R. Mamy, M. Goiran, A. R. Fert, C. Armand, J, C. Ousset, J. Magn. Magn. Mater. 156, 197-198 (1996).

10. Labrune M., Andrieu S., Rio F., and Bernstein P. Time dependence of the magnetization process of re-tm alloys. J. Magn. Magn. Mater. 80:211, (1989).

11. Fatuzzo E. Theoretical considerations on the switching transient in ferroelectrics. Phys. Rev., 127(6):1999, (1962).

12. H. Ardhuin, E. Snoeck et M.J. Casanove, J. Crystal Growth, 182, 394 (1987).

13. A. Adanlété Adjanoh, R. Belhi, J. Vogel, O. Fruchart, M. Ayadi, K. Abdelmoula, Journal of Magnetism and Magnetic Materials 322, 2498 (2010).

14. C. Chappert and P. Bruno, J. Appl. Phys. 64, 5736 (1988).

15. P. Bruno, G. Bayreuther, C. Chappert, G. Lugert, D. Renard, and J. Seiden, J. Appl. Phys. 68, 5759 (1990). 